Case report

\title{
Approaching cavitary lung lesion in a patient with pulmonary embolism
}

\author{
Aurelia Crețu ${ }^{*, 1,2}$, Elena Cristina Mitrofan ${ }^{3}$
}

1Department of Pharmacology, Clinical Pharmacology and Algesiology, "Grigore T. Popa" University of Medicine and Pharmacy, Iași, Romania; ${ }^{2}$ Regional Institute of Oncology, Iași, Romania; ${ }^{3}$ Clinical Pneumophtisiology Hospital, laşi, Romania

\begin{abstract}
The pulmonary cavity may reflect the presence of a broad spectrum of lung pathological processes, with surprising etiology at times. Bacteria, fungi, viruses, parasites, embolism, malignancy, and autoimmune disease may be involved in its formation. We present the case of a 78 year-old patient who was hospitalized 7 days prior to admission to our hospital with grade IV mMRC dyspnea and dry cough, the chest CT indicating pulmonary embolism and the presence of a pulmonary cavity, suggestive of neoplastic etiology. An infection with Staphylococcus aureus complicated with pulmonary embolism, two pathological entities which can lead to cavitary pulmonary damage, can prove challenging to any physician in the given context.
\end{abstract}

Keywords: lung cavity; Staphylococcus aureus; pulmonary embolism

\section{Introduction}

The cavity, a gas filled space in a pulmonary consolidation area, mass or nodule, is produced by the expulsion of the necrotic content in a bronchus. It may or may not contain a liquid level and is surrounded by a wall of variable thickness, being a manifestation of a broad spectrum of lung pathological processes [1].

A rather rare finding in pulmonary imaging, the cavity formation may result from bacterial infections such as microaerophilic streptococci, viridans streptococci, Klebsiella pneumoniae, Staphylococcus aureus, Pseudomonas aeruginosa, Haemophilus influenzae, Acinetobacter species, Escherichia coli, Legionella species, Fusobacterium necrophorum, Nocardia asteroides,

Received: July 2018; Accepted after review: December 2018; Published: December 2018.

${ }^{*}$ Corresponding author: Aurelia Crețu, Department of Pharmacology, Clinical Pharmacology and Algesiology, "Grigore T. Popa" University of Medicine and Pharmacy, 16 Universității Str, Iași, Romania.

Email: aureliabaciucretu@gmail.com mycobacteria such as Mycobacterium tuberculosis, nontuberculous mycobacteria and Mycobacterium avium complex, fungi such as Cryptococcus neoformans, Coccidioides, Aspergillus and mucormycosis, parasites such as Paragonimus westermani and Echinococcus genus, and viral infections such as papilloma virus [1,2]. Moreover, the cavity may be the result of a septic pulmonary embolism, such as Lemierre's syndrome, or a primary or secondary malignancy (lung cancer, lymphoma, squamous cell carcinoma, sarcoma), autoimmune disease (rheumatoid arthritis, granulomatosis with polyangiitis, ankylosing spondylitis, histiocytosis) [1-5].

We present the case of a patient with cavitary pulmonary disease on plain chest radiography, an entity with a challenging diagnosis in the context of rich pathological history and significant family history.

\section{Case report}

A 78-year-old non-smoker female patient was transferred to our clinic from a regional 
hospital with the suspicion of a right bronchopulmonary neoplasm. The patient presented to the hospital seven days prior to admission in our clinic with dry cough and sudden onset, grade IV on the modified Medical Research Council (mMRC) dyspnea scale, fatigue and palpitations; she was hospitalized in the cardiology department for massive bilateral pulmonary embolism confirmed by computed tomography (CT), followed by a treatment with intravenous heparin in continuous infusion. The patient was then transferred to our department in an influenced, but stable state. There was no recorded history of fever, hemoptysis, weight loss, tuberculosis exposure or venous thrombosis prior to admission.

The patient has a personal history of malignancy, breast cancer, for which the patient underwent with chemo- and radiotherapy and surgery three years prior the current admission, uterine fibromatosis operated six years before - total hysterectomy and bilateral oophorectomy), with no relapse of breast cancer and no hormonal treatment. The complex cardiovascular pathology included past paroxystic atrial fibrillation, ventricular extrasystolic arrhythmia, aortic atherosclerosis, NYHA class II heart failure, grade III tricuspid regurgitation, grade I mitral regurgitation, low pulmonary hypertension, previously treated with propafenone/ amiodarone and acetylsalicylic acid, currently under treatment with bisoprolol $2.5 \mathrm{mgX} 2 /$ day. She had 5 pregnancies, only one delivered on term, the others recorded as spontaneous abortions. Her family history highlighted the death of her father at the age of 66 due to broncho-pulmonary neoplasm, two deceased brothers with lung cancer and a sister deceased probably due to cardiovascular complications.

Upon admission, patient's clinical examination revealed influenced general state, pallor, multiple bruises on the upper limbs, abdomen and a second grade decubitus ulcer on the right external malleolus. On inspection, two straight post-quadrantectomy scars were present on the anterior side of the right hemithorax. Her breathing rate was 15 breaths/minute, with dullness of the basal right hemithorax on percussion and abolished vesicular breathing sounds at the base of the right hemithorax on auscultation. The saturation of peripheral oxygen (SpO2) was $92 \%$. Recorded cardiac rhythm normal, at 80 beats/minute and the blood pressure was $108 / 62 \mathrm{mmHg}$. On admission, the performed lab tests included a white blood cell count of $10480 / \mu \mathrm{L}$, erythrocyte sedimentation rate (ESR) of $140 \mathrm{~mm} / 1$ hour, thrombocytosis of $525000 / \mu \mathrm{L}$ and normocytic normochromic anemia with $\mathrm{Hb}$ of $10 \mathrm{~g} / \mathrm{dl}$, urea of $10,1 \mathrm{mg} / \mathrm{dL}$, creatinine of $0,55 \mathrm{mg} / \mathrm{dL}$, glycemia of 89,1 $\mathrm{mg} / \mathrm{dL}$, ALAT of $19,5 \mathrm{U} / \mathrm{L}$, ASAT of $19,6 \mathrm{U} / \mathrm{L}$, total proiein count of $69,26 \mathrm{~g} / \mathrm{L}$ were also recorded. The patient had previously received antibiotic treatment with amoxicillin / acidum clavulanicum $1.2 \mathrm{~g}$ every $12 \mathrm{~h}$ for seven days.

The chest CT revealed lesions suggestive of pulmonary embolism in the distal segments of the right pulmonary artery and the proximal left inferior lobar artery, a cavitary image with anfractuous wall in the upper right lobe, right basal pleural fluid (Figure 1). Vertebral bone hemangiomas were identified at D7 and D8 level.

The chest radiograph on admission revealed an area of hyperlucency containing air-fluid level in the lower $1 / 3$ of the right lung area and an area of opacity in the lower $1 / 2$ of the right hemithorax, with concave superior interface border (Figure 2).

The pleural ultrasound performed on the second day of admission showed a finely septated pleural effusion of aproximmately 3.5 $\mathrm{cm}$ depth, which maintained the same aspect until the 13th day of hospitalization. Diagnostic thoracentesis was performed, revealing an exudate of uncertain origin.

The ear, nose and throat examination performed on second day of admission revealed paresis of the left recurrent laryngeal nerve, with no identifiable primary upper respiratory tract tumor. 


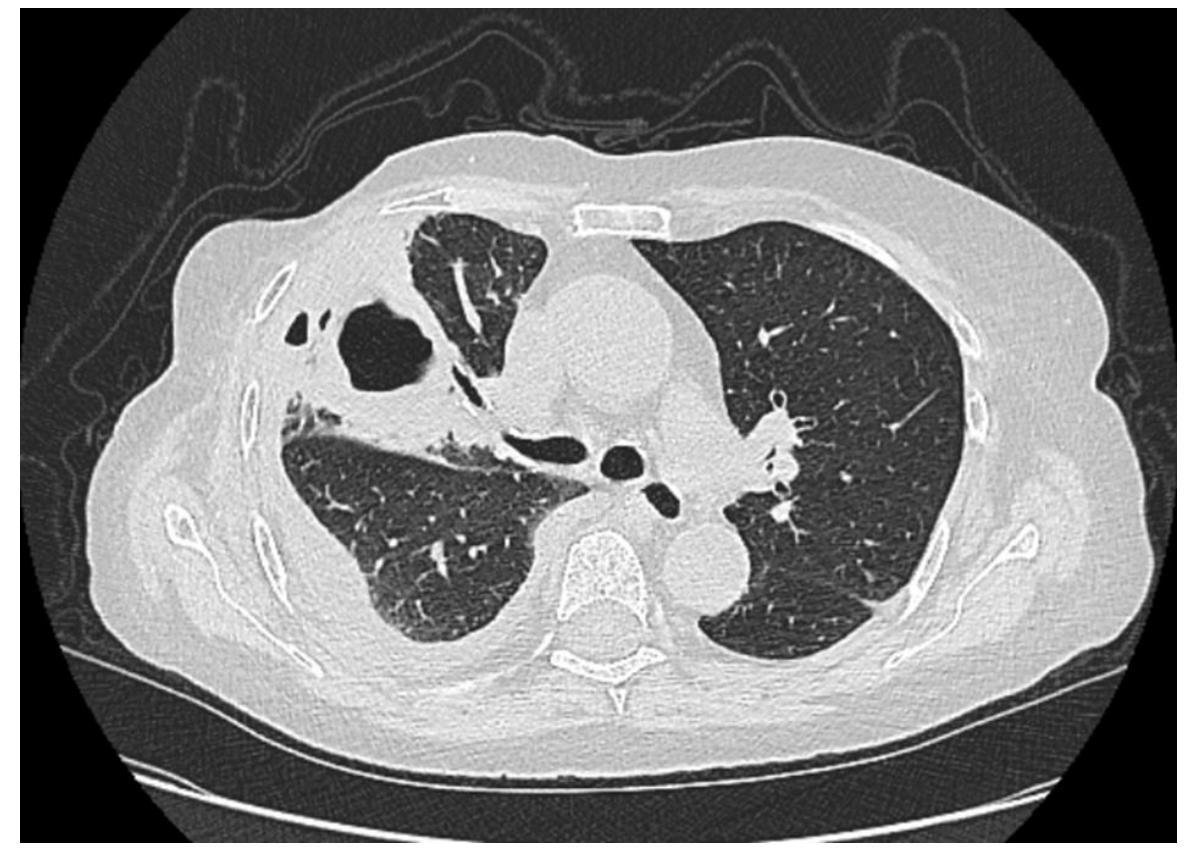

Fig. 1. CT scan image showing a thick-walled cavity. Right basal pleural fluid.

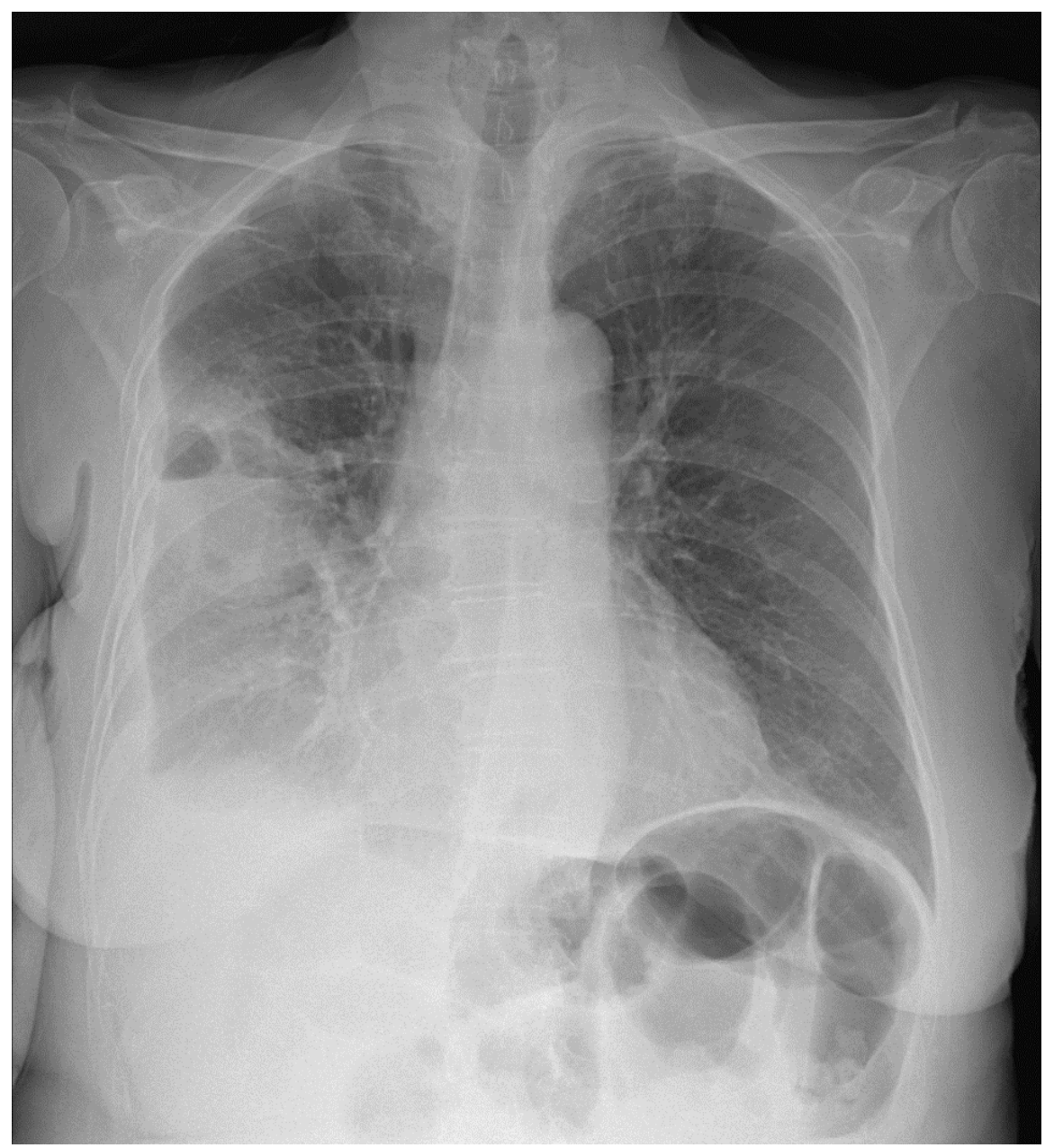

Fig 2. Hyperlucent area containing an air-fluid level in lower $1 / 3$ of the right lung and chest opacity in lower $1 / 2$ of right hemithorax, with concave superior interface border. 
The fibro-bronchoscopy examination revealed purulent secretions of white-viscous appearance adherent to the bronchial mucosa in the upper right lobe, probably from the middle lobe or basal pyramid. Samples were sent to the laboratory for cultures on specific media for common bacteria, Mycobacterium tuberculosis and fungi. Bronchial aspiration cytology noted the presence of a moderate inflammatory reaction with neutrophils, Gram positive bacteria, with cultures revealing methicillin-sensitive Staphylococcus aureus (3+). The patient's antibiotic therapy was then adapted according to the antibiogram. She was started with $1 \mathrm{~g}$ of ceftriaxone every 8 hours (intravenous administration) and $500 \mathrm{mg}$ of metronidazole every 6 hours (per os) for five days, later switched on $600 \mathrm{mg}$ of clindamycin every 8 hours (intravenous adminsitration) for 10 days, followed by per os administration for two weeks after discharge.

The patient's condition improved slowly. Under targeted antibiotic regimen, oxygen therapy, low molecular weight heparin therapy, cough supressants, and analgesia, her clinical, biological and imaging parameters steadily improved.

With adequate wound dressing and topical cutaneous creams, the decubitus ulcer healed. Follow-up examination revealed the following parameters: white blood cell count of $7010 / \mu \mathrm{L}$, hemoglobin of $12.7 \mathrm{~g} / \mathrm{dL}$, platelet count of $323000 / \mu \mathrm{L}$, ESR of $88 \mathrm{~mm} / 1$, urea of 18.6 $\mathrm{mg} / \mathrm{dL}$, creatinine of $0.52 \mathrm{mg} / \mathrm{dL}$, glycemia of $109 \mathrm{mg} / \mathrm{dL}$, ALAT of $12.5 \mathrm{U} / \mathrm{L}$, ASAT of $7 \mathrm{U} / \mathrm{L}$, total protein count of $75 \mathrm{~g} / \mathrm{L}$.

She was discharged of the $15^{\text {th }}$ day of hospitalization with recommendations for two weeks of antibiotic therapy, continuous anticoagulation and regular check-ups. The follow-up at one, two and three months showed steady improvement, confirmed by imaging studies. Chest $x$-ray showed the accentuation of the peribronchovascular interstitum in $1 / 3$ of the right hemithorax at three months (Figure 3 ) and the persistence of a lower left lobe arterial thrombus, with complete medium lobe artery obstruction on CT scan.

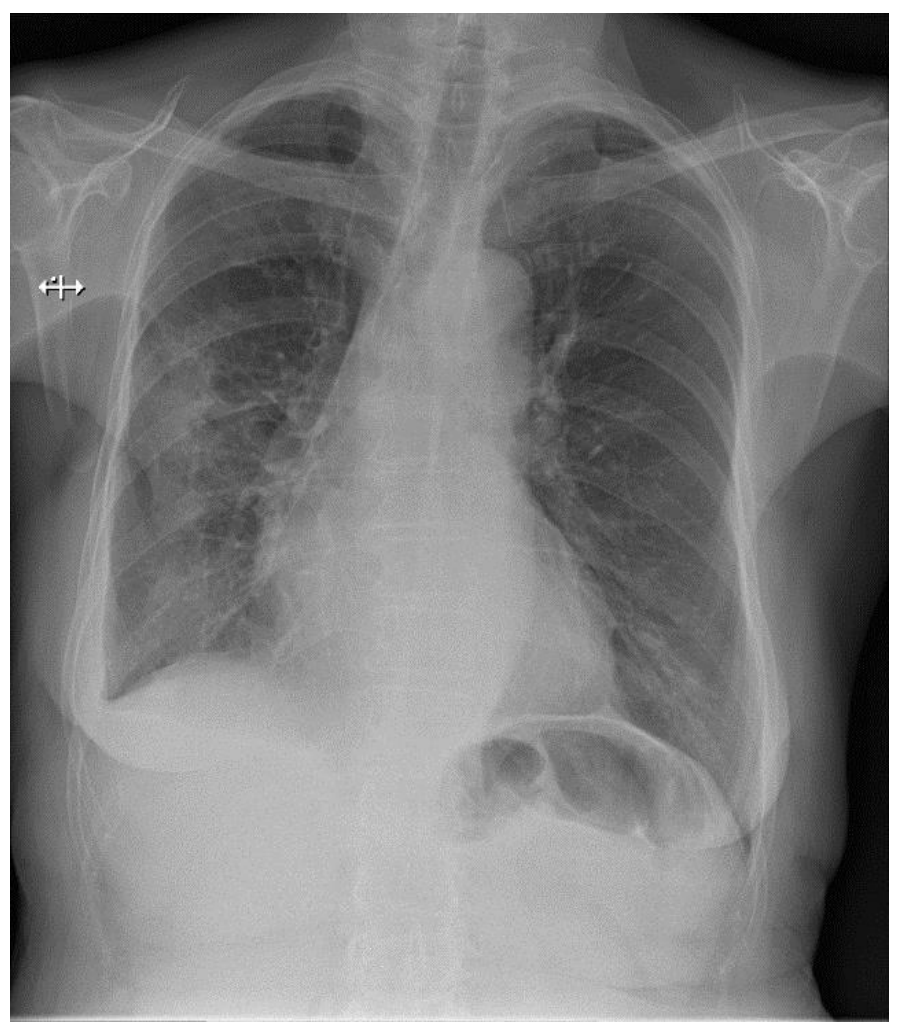

Fig. 3. Chest radiography at three months. Accentuating peribronchovascular interstitium $1 / 3$ of the right lung area. 


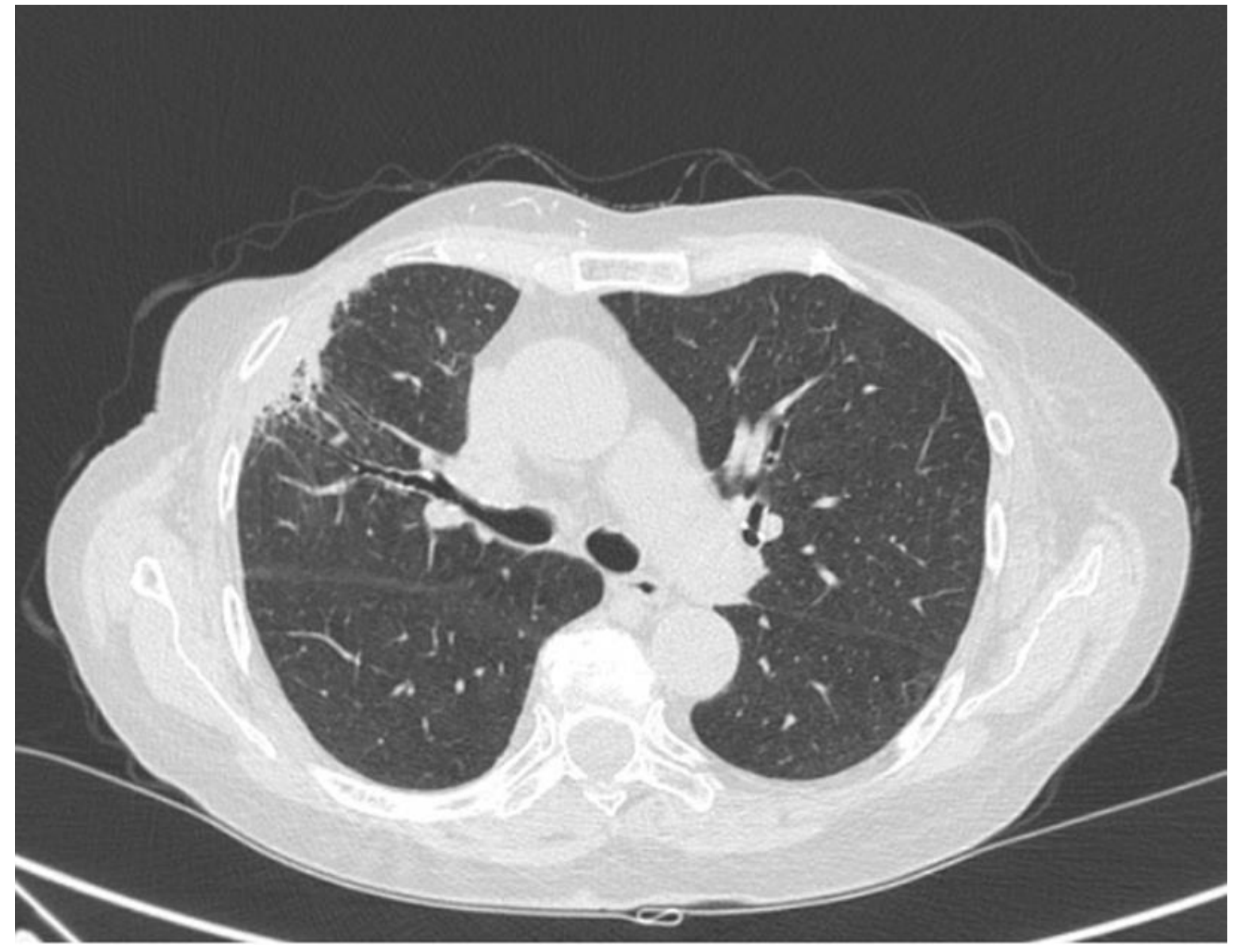

Fig. 4. Chest CT scan at three months. Resolution of cavity. Right pachypleuritis.

The topography of the former abscess showed a small residual air-filled cavity communicating with the bronchial tree and bronchiectasis in the medium lobe. Groundglass appearance at the level of the left basal pyramid and the right posterior basal segment were observed (Figure 4). At three months, the blood parameters showed normal complete blood count, ESR of $47 \mathrm{~mm} / 1 \mathrm{~h}$, normal renal and liver function. At seven months after discharge the patient's general condition significantly improved.

\section{Discussions}

In regards to cavitary pulmonary disease, although radiological imaging may be highly suggestive of a diagnosis, only through integrating the clinical context and targeted paraclinical investigations can one achieve a complete and correct diagnosis.

Before approaching a cavitary lesion, clinicians must exclude the great mimics of such an entity, such as cysts, emphysema bullae, infected lung bullae, cystic bronchiectasis [1, 2].

Recent studies propose an algorithm to address cavitary pulmonary disease, in respect to the duration of clinical symptomatology and the radiological findings, as well as the supplementary investigations. Thus, acute and subacute cavities $\quad<12$ weeks) may be determined by common bacteria that produce lung abscess, necrotizing pneumonia, septic emboli and fungal infections [2].

Hemocultures in patients with septic emboli frequently highlight staphylococcus, Fusobacterium necrophorum, Klebsiella pneumoniae and Streptococcus viridans. Among the more common fungal infections present, literature notes the ones caused by Cryptococcus neoformans and Aspergillus species.

When facing a lesion such as an acute cavity, clinicians should always take a detailed history, accompanied by parameters such as a 
complete blood count, procalcitonin, blood culture, sputum culture and integrate the resulting data with the CT scan features of the lesion. The same conduct applies in the case of a chronic cavity, albeit a biopsy should be done if the complete diagnosis remains unclear [2].

Chronic cavities (> 12 weeks) can be determined by three categories of disease: chronic infection, malignancy and autoimmune disease. Chronic infections include Mycobacterium tuberculosis, nontuberculous mycobacteria (with a particular risk group such as elderly women without structural pulmonary disease), chronic necrotic aspergillosis, histoplasmosis, blastomycosis, paragomiasis, echinococcosis and tracheobronchial papillomatosis. A cavity wall $>15 \mathrm{~mm}$ thick on chest X-ray is highly suggestive for malignancies (in $90 \%$ of the cases) $[1,4,6]$. The autoimmune disease most likely leading to the formation of a pulmonary cavitary lesion is granulomatosis with polyangeitis, with 40$70 \%$ of patients having nodular lung lesions and $25-50 \%$ of these cases, in which nodules are $>2 \mathrm{~cm}$ presenting with cavities. In $20 \%$ of patients with rheumatoid arthritis, imaging studies highlight lung rheumatoid nodules, which may develop central necrosis. The main differential diagnosis in all mentioned cases is lung cancer $[2,5]$.

The patient history for the presented case raised the suspicion of an acute cavity, although the diagnosis of bronchopulmonary neoplasm could not be initially ruled out. Staphylococcus aureus is rarely isolated in cultures from such lesions; it may be found in patients with a certain background, and causing necrotizing pneumonia, a rare but very severe diagnosis with some cases originating from uncomplicated skin and softtissue infections [7, 8]. The most prevalent microorganisms isolated are Haemophilus influenzae and Streptococcus pneumonia followed by Klebsiella pneumonia, Pseudomonas aeruginosa. Community acquired pneumonias and cavities caused by Staphylococcus aureus are uncommon and usually occur after influenza or from staphylococcal bacteremia [9-11]. In our case, a possible source of can point in the direction of the decubitus ulcer, from which samples for culture were not taken.

A non-malignant cavitary lesion can mimic malignant lesion on most radiological examinations. Also, for pulmonary thromboembolism, the causes are various, including a state of hypercoagulability induced by sepsis. Virchows' triad suggests that the pathogenesis of venous thrombosis consists of stasis (stagnant flow), vessel wall damage, and disorders of coagulation (hypercoagulability). Damage to vessel wall can be due to infection, infiltration or trauma. However, the more common issues are coagulation defects that cause a hypercoagulability state which can result in sudden death from silent emboli [12].

The three month follow-up CT scan confirmed one of our hypotheses, namely the presence of bronchiectasis, which is a predisposing factor for pneumonitis. Appropriate long-term treatment was indicated. Anticoagulant therapy initiated by continuous intravenous infusion was replaced with subcutaneous treatment, required for lifetime. At the six month follow-up, the presence of thrombophilia (factor $\mathrm{V}$ Leiden, plasma antithrombin, protein $\mathrm{C}$, and protein $\mathrm{S}$ activity measurements) can be assessed, given the patient's history. However, further findings will not change the current treatment or prognosis of the patient [13].

\section{Conclusions}

Identifying the etiology of a cavitary pulmonary disease is a challenge due to the wide range of factors and large variety of clinical manifestations. A particular diagnosis can be favored in the context of clinical and radiological data, but the patient must always be fully investigated, following all diagnostic paths in order to exclude other causes.

\section{Consent}

Written informed consent was obtained from the patient for publication of this case report.

\section{Competing interests}

The authors declare that they have no competing interests. 


\section{References}

1. Gadkowski LB, Stout JE. Cavitary pulmonary disease. Clin Microbiol Rev 2008; 21(2):305333.

2. Gafoor K, Patel S, Girvin F, et al. Cavitary lung diseases - A clinical-radiological algorithmic approach. Chest 2018; 153(6):1443-1465.

3. Gerada E, Gatt N, Mizzi A, Montefort S. Atypical cavitary lung lesions: a case report and review of radiologic manifestations. Intern Med 2014; 4:131.

4. Gill RR, Matsusoka S, Hatabu H. Cavities in the lung in oncology patients: imaging overview and differential diagnoses. Appl Radiol 2010; 39(6):10-21.

5. Mayberry JP, Primack SL, Müller NL. Thoracic manifestations of systemic autoimmune diseases: radiographic and high-resolution CT findings. Radio Graphics 2000; 20(6):16231635.

6. Serrano Usaola N, Martin Egaña M, Beltran de Otalora Garcia S, et al. Cavitated lung lesions. A diagnostic approach. European Society of Radiology ECR 2013/C-0351 2013; 1-24. Doi: 10.1594/ecr2013/C-0351.

7. Brown ML, O'Hara FP, Close NM, et al. Prevalence and sequence variation of pantonvalentine leukocidin in methicillin-resistant and methicillin-susceptible Staphylococcus aureus strains in the United States. J Clin. Microbiol 2012; 50(1):86-90.
8. Chatha N, Fortin D, Bosma KJ. Management of necrotizing pneumonia and pulmonary gangrene: $A$ case series and review of the literature. Can Respir J 2014; 21(4):239-245.

9. Chou DW, Wu SL, Chung KM, Han SC, Cheung BM. Septic pulmonary embolism requiring critical care: clinicoradiological spectrum, causative pathogens and outcomes. Clinics 2016; 71(10):562-569.

10. Manafi A, Khodabandehloo M, Rouhi S, et al. Molecular epidemiology survey of Staphylococcus aureus panton-valentine leukocidin-positive isolated from Sanandaj, Iran. Adv Biomed Res 2017; 6:87.

11. leven M, Coenen S, Loens K, et al. Aetiology of lower respiratory tract infection in adults in primary care: a prospective study in 11 European countries. Clin Microbiol Infect 2018; 1.e1-1.e6.

12. Kumar DR, Hanlin E, Glurich I, Mazza JJ, Yale $\mathrm{SH}$. Virchow's contribution to the understanding of thrombosis and cellular biology. Clin Med Res 2010; 8(3-4):168-172.

13. Méan $M$, Limacher $A$, Stalder $O$, et al. Do Factor V Leiden and Prothrombin G20210A mutations predict recurrent venous thromboembolism in older patients? Am J Med 2017; 130(10):1220.e17-1220.e22. 